PROCEEDINGS OF THE

AMERICAN MATHEMATICAL SOCIETY

Volume 134, Number 9, September 2006, Pages 2559-2569

S 0002-9939(06)08260-8

Article electronically published on February 8, 2006

\title{
SPECTRA OF ALGEBRAS OF ENTIRE FUNCTIONS ON BANACH SPACES
}

\author{
ANDRIY ZAGORODNYUK
}

(Communicated by N. Tomczak-Jaegermann)

\begin{abstract}
We obtain an explicit description of the spectrum (set of closed maximal ideals) of $H_{b}(X)$, algebra of analytic functions on a Banach space $X$ which are bounded on bounded subsets. We show that the spectrum of $H_{b}(X)$ admits a natural linear structure. Some applications to the algebra of uniformly continuous and bounded analytic functions on the unit ball $B \subset X$ are indicated.
\end{abstract}

Let $A$ be a complex commutative topological algebra. Let us denote by $M(A)$ the spectrum (set of closed maximal ideals $=$ set of continuous characters $=$ set of continuous complex-valued homomorphisms) of $A$. Recall that $A$ is semisimple if the complex homomorphisms from $M(A)$ separate points of $A$. It is well known that every semisimple commutative Fréchet algebra $A$ is isomorphic to some subalgebra of continuous functions on $M(A)$ endowed with a natural topology. More exactly, for every $a \in A$ there exists a function $\widehat{a}: M(A) \rightarrow \mathbb{C}$ defined by $\widehat{a}(\phi):=\phi(a)$. The weakest topology on $M(A)$ such that all functions $\widehat{a}, a \in A$, are continuous is called the Gelfand topology. The Gelfand topology coincides with the weak-star topology of the strong dual space $A^{\prime}$, restricted to $M(A)$. If $A$ is a Banach algebra, $M(A)$ is a weak-star compact subset of the unit ball of $A^{\prime}$.

If $A$ is a uniform algebra of continuous functions on a metric space $G$, then for any $x \in G$ the point evaluation functional $\delta(x): f \mapsto f(x)$ belongs to $M(A)$.

The purpose of this paper is to describe the spectrum of the Fréchet algebra $H_{b}(X)$ of entire analytic functions of bounded type on a Banach space $X$ and to study some related questions of infinite-dimensional holomorphy.

The problem of description of the spectrum of $H_{b}(X)$ was first studied by Aron, Cole and Gamelin 3, 4. Using the Aron-Berner extension operation 2, 10, they showed, in particular, that $X^{\prime \prime}$ belongs to the spectrum of $H_{b}(X)$. In [5] it is proved that this inclusion is proper if there exists a polynomial on $X$ which is not weakly continuous on bounded sets. This approach was generalized for algebra-valued analytic functions by García et al. in [18. Some analytic structure on the set of maximal ideals was considered in [5] (for generalization for algebra-valued functions see [17). In 22 Mujica investigated ideals of analytic functions of bounded type on

Received by the editors July 8, 2004 and, in revised form, January 28, 2005, February 1, 2005, and March 15, 2005.

2000 Mathematics Subject Classification. Primary 46J15, 46J20; Secondary 46E15, 46E25, 46G20, 46G25.

Key words and phrases. Infinite-dimensional holomorphy, spectra of algebras.

This research was supported in part by NSERC research grant OGP 005616.

(C)2006 American Mathematical Society Reverts to public domain 28 years from publication 
Tsirelson's space $T$ and showed that each character on $H_{b}(T)$ is a point evaluation functional. Homomorphisms of $H_{b}$ were studied by Carando, García and Maestre in [9]. In 1] Alencar et al. considered maximal ideals of algebras of symmetric analytic functions on $\ell_{p}$.

In this paper we show that every element of the spectrum of $H_{b}(X)$ can be represented by a sequence of functionals $\left(u_{k}\right)_{k=1}^{\infty}$ such that each $u_{k}$ belongs to a Banach space $E_{k}$, where $E_{1}=X^{\prime \prime}$ and $E_{n}$ coincides with a special subspace of linear functionals on $n$-homogeneous polynomials. It is also shown that the spectrum of $H_{b}(X)$ contains the linear space of all finite sequences $\left(u_{1}, \ldots, u_{m}, 0,0, \ldots\right)$. Finally, some related examples are considered.

For background on analytic functions on infinite-dimensional spaces, we refer the reader to [13] or to 21]. For details on the Aron-Berner extension we refer to [8].

For a given complex Banach space $X, \mathcal{P}\left({ }^{n} X\right)$ (resp. $\left.\mathcal{P}\left({ }^{\leq n} X\right)\right)$ denotes the Banach space of all continuous $n$-homogeneous complex-valued polynomials on $X$ (resp. the Banach space of all continuous $n$-degree complex-valued polynomials on $X$ ). $\mathcal{P}_{f}\left({ }^{n} X\right)$ denotes the subspace of $n$-homogeneous polynomials of finite type, that is the subspace generated by all polynomials of the form $P(x)=(\gamma(x))^{n}$ with $\gamma \in X^{\prime}$ and $\mathcal{P}_{c}\left({ }^{n} X\right)$ is the closure of $\mathcal{P}_{f}\left({ }^{n} X\right)$ with the topology of uniform convergence on bounded subsets of $X$. It is well known [6] that if $X^{\prime}$ has the approximation property, then $\mathcal{P}_{c}\left({ }^{n} X\right)$ coincides with $\mathcal{P}_{w u}\left({ }^{n} X\right)$, the space of $n$-homogeneous polynomials which are weakly uniformly continuous on bounded subsets of $X$.

Recall that for every polynomial $P \in \mathcal{P}\left({ }^{n} X\right)$ there exists a (necessarily unique) symmetric $n$-linear form $A_{P}$, associated with $P$ such that $A_{P}(x, \ldots, x)=P(x)$. We will write $A_{P}\left(x_{1}^{k_{1}}, \ldots, x_{n}^{k_{n}}\right)$ instead of $A_{P}(\underbrace{x_{1}, \ldots, x_{1}}_{k_{1}}, \ldots, \underbrace{x_{n}, \ldots, x_{n}}_{k_{n}})$. We will use the fact that $\mathcal{P}\left({ }^{n} X\right)$ is isomorphic to the dual space of the symmetric projective $n$-fold tensor product $\bigotimes_{s, \pi}^{n} X$ of $X$.

Let us denote by $A_{n}(X)$ the closure of the algebra, generated by polynomials from $\mathcal{P}\left({ }^{\leq n} X\right)$ with respect to the uniform topology on bounded subsets. It is clear that $A_{1}(X) \cap \mathcal{P}\left({ }^{n} X\right)=\mathcal{P}_{c}\left({ }^{n} X\right)$ and $A_{n}(X)$ is a Fréchet algebra of entire analytic functions on $X$ for every $n$. The closure of the algebra of all polynomials $\mathcal{P}(X)$ with respect to the uniform topology on bounded subsets is denoted by $H_{b}(X)$ and is called the algebra of entire functions of bounded type on $X$. It is well known that $H_{b}(X)$ consists of all entire functions that are bounded on bounded subsets. The closure of the algebra of all polynomials with respect to the uniform topology on the unit ball $B, H_{u c}^{\infty}(B)$, is the algebra of all analytic functions on $B$ which are uniformly continuous and bounded. We will use the short notation $M_{b}$ and $M_{u c}$ for the spectra $M\left(H_{b}(X)\right)$ and $M\left(H_{u c}^{\infty}(B)\right)$ respectively.

According to [3], every continuous functional $\phi \in H_{b}(X)^{\prime}$ can be represented by $\phi=\sum_{k=0}^{\infty} \phi_{k}$, where $\phi_{k}=\pi_{k}(\phi)$ is the restriction of $\phi$ to $\mathcal{P}\left({ }^{k} X\right)$. The infimum of all $r>0, R(\phi)$ such that $\phi$ is continuous with respect to the norm of uniform convergence on the ball $r B$ is called the radius function of $\phi$. It is known [3] that

$$
R(\phi)=\limsup _{n \rightarrow \infty}\left\|\phi_{n}\right\|^{1 / n}
$$

For every polynomial $P \in \mathcal{P}\left({ }^{m k} X\right)$ we denote by $P_{(m)}(u)$ the polynomial from $\mathcal{P}\left({ }^{k} \bigotimes_{s, \pi}^{m} X\right)$ such that $P_{(m)}\left(x^{\otimes m}\right)=P(x)$, where $x^{\otimes m}=\underbrace{x \otimes \cdots \otimes x}_{m \text { times }}$. 
Lemma 1. Let $\phi \in H_{b}(X)^{\prime}$ such that $\phi(P)=0$ for every $P \in \mathcal{P}\left({ }^{m} X\right) \cap A_{m-1}(X)$, where $m$ is a fixed positive integer and $\phi_{m} \neq 0$. Then there is $\psi \in M_{b}$ such that $\psi_{k}=0$ for $k<m$ and $\psi_{m}=\phi_{m}$. The radius function $R(\psi)=\left\|\phi_{m}\right\|^{1 / m}$.

Proof. Since $\phi_{m} \neq 0$, there is an element $w \in\left(\bigotimes_{s, \pi}^{m} X\right)^{\prime \prime}, w \neq 0$ such that for any $m$-homogeneous polynomial $P, \phi(P)=\phi_{m}(P)=\widetilde{P}_{(m)}(w)$, where $\widetilde{P}_{(m)}$ is the Aron-Berner extension of the linear functional $P_{(m)}$ from $\bigotimes_{s, \pi}^{m} X$ to $\left(\bigotimes_{s, \pi}^{m} X\right)^{\prime \prime}$ and $\|w\|=\left\|\phi_{m}\right\|$. For an arbitrary $n$-homogeneous polynomial $Q$ we set

$$
\psi(Q)=\left\{\begin{array}{lr}
\widetilde{Q}_{(m)}(w) & \text { if } n=m k \text { for some } k \geq 0 \\
0 & \text { otherwise }
\end{array}\right.
$$

where $\widetilde{Q}_{(m)}$ is the Aron-Berner extension of the $k$-homogeneous polynomial $Q_{(m)}$ from $\bigotimes_{s, \pi}^{m} X$ to $\left(\bigotimes_{s, \pi}^{m} X\right)^{\prime \prime}$.

Let $\left(u_{\alpha}\right)$ be a net from $\bigotimes_{s, \pi}^{m} X$ that converges to $w$ in the weak-star topology of $\left(\bigotimes_{s, \pi}^{m} X\right)^{\prime \prime}$, where $\alpha$ belongs to an index set $\mathfrak{A}$. We can assume that $u_{\alpha}$ has a representation $u_{\alpha}=\sum_{j=1}^{\infty} x_{j, \alpha}^{\otimes m}$ for some $x_{j, \alpha} \in X$. Let us show that $\psi(P Q)=$ $\psi(P) \psi(Q)$ for any homogeneous polynomials $P$ and $Q$. Let us suppose first that $\operatorname{deg}(P Q)=m r+l$ for some integers $r \geq 0$ and $m>l>0$. Then $P$ or $Q$ has degree equal to $m k+s, k \geq 0, m>s>0$. Thus, by the definition, $\psi(P Q)=$ 0 and $\psi(P) \psi(Q)=0$. Suppose that $\operatorname{deg}(P Q)=m r$ for some integer $r \geq 0$. If $\operatorname{deg} P=m k$ and $\operatorname{deg} Q=m n$ for $k, n \geq 0$, then $\operatorname{deg}(P Q)=m(k+n)$ and $\psi(P Q)=$ $(\widetilde{P Q})_{(m)}(w)=\widetilde{P}_{(m)}(w) \widetilde{Q}_{(m)}(w)=\psi(P) \psi(Q)$.

Now let $\operatorname{deg} P=m k+l$ and $\operatorname{deg} Q=m n+r, l, r>0, l+r=m$. Write $\nu=$ $1 /(\operatorname{deg} P+\operatorname{deg} Q) !=1 /(m(k+n+1)) !$. Let $A_{P Q}$ denote the symmetric multilinear map, associated with $P Q$. Then

$$
\begin{aligned}
& A_{P Q}\left(x_{1}, \ldots, x_{m(k+n+1)}\right) \\
& =\nu \sum_{\sigma \in S_{m(k+n+1)}} A_{P}\left(x_{\sigma(1)}, \ldots, x_{\sigma(m k+l)}\right) A_{Q}\left(x_{\sigma(m k+l+1)}, \ldots, x_{\sigma(m(k+n+1))}\right),
\end{aligned}
$$

where $S_{m(k+n+1)}$ is the group of permutations on $\{1, \ldots, m(k+n+1)\}$. Thus for $\alpha_{1}, \ldots, \alpha_{k+n+1} \in \mathfrak{A}$ we have

$$
\begin{aligned}
\psi(P Q) & =(\widetilde{P Q})_{(m)}(w)=\lim _{\alpha_{1}, \ldots, \alpha_{k+n+1}} \widetilde{A}_{P Q_{(m)}}\left(u_{\alpha_{1}}, \ldots, u_{\alpha_{k+n+1}}\right) \\
& =\lim _{\alpha_{1}, \ldots, \alpha_{k+n+1}} \widetilde{A}_{P Q_{(m)}}\left(\sum_{j=1}^{\infty} x_{j, \alpha_{1}}^{\otimes m}, \ldots, \sum_{j=1}^{\infty} x_{j, \alpha_{k+n+1}}^{\otimes m}\right) \\
& =\nu \sum_{\sigma \in S_{m(k+n+1)}} \lim _{\alpha_{\sigma(1)}, \ldots, \alpha_{\sigma(k+n+1)}} \sum_{j_{1}, \ldots, j_{k+n+1}=1}^{\infty} A_{P}\left(x_{j_{\sigma(1)}, \alpha_{\sigma(1)}}^{m}, \ldots, x_{j_{\sigma(k)}, \alpha_{\sigma(k)}}^{m},\right. \\
& \left.x_{j_{\sigma(k+1)}, \alpha_{\sigma(k+1)}}^{l}\right) A_{Q}\left(x_{j_{\sigma(k+1)}^{r}, \alpha_{\sigma(k+1)}}^{r}, x_{j_{\sigma(k+2)}, \alpha_{\sigma(k+2)}}^{m}, \ldots, x_{\left.j_{\sigma(k+n+1)}, \alpha_{\sigma(k+n+1)}\right)}^{m}\right) .
\end{aligned}
$$

Fix some $\sigma \in S_{m(k+n+1)}$ and fix all $x_{j_{\sigma(i)}, \alpha_{\sigma(i)}}$, for $i \leq k$ and for $i>k+1$. Then

$$
\begin{aligned}
& \sum_{j_{1}, \ldots, j_{k+n+1}=1}^{\infty} \lim _{\alpha_{\sigma(k+1)}} A_{P}\left(x_{j_{\sigma(1)}, \alpha_{\sigma(1)}}^{m}, \ldots, x_{j_{\sigma(k)}, \alpha_{\sigma(k)}}^{m}, x_{j_{\sigma(k+1)}, \alpha_{\sigma(k+1)}}^{l}\right) \\
& \times A_{Q}\left(x_{j_{\sigma(k+1)}^{r}, \alpha_{\sigma(k+1)}}^{r}, x_{j_{\sigma(k+2)}, \alpha_{\sigma(n+2)}}^{m}, \ldots, x_{j_{\sigma(k+n+1)}, \alpha_{\sigma(k+n+1)}}^{m}\right)=0
\end{aligned}
$$


because for fixed $x_{k_{\sigma(i)}, \alpha_{\sigma(i)}}, i \leq k$,

$$
P_{\sigma}(y):=\sum_{j_{1}, \ldots, j_{k}, j_{k+2}, \ldots, j_{k+n+1}=1}^{\infty} A_{P}\left(x_{j_{\sigma(1)}, \alpha_{\sigma(1)}}^{m}, \ldots, x_{j_{\sigma(k)}, \alpha_{\sigma(k)}}^{m}, y^{l}\right)
$$

is an $l$-homogeneous polynomial and for fixed $x_{k_{\sigma(i)}, \alpha_{\sigma(i)}}, i>k+1$,

$$
Q_{\sigma}(y):=\sum_{j_{1}, \ldots, j_{k}, j_{k+2}, \ldots, j_{k+n+1}=1}^{\infty} A_{Q}\left(y^{r}, x_{j_{\sigma(k+2)}, \alpha_{\sigma(n+2)}}^{m}, \ldots, x_{j_{\sigma(k+n+1)}, \alpha_{\sigma(k+n+1)}}^{m}\right)
$$

is an $r$-homogeneous polynomial. Thus $P_{\sigma} Q_{\sigma} \in A_{m-1}(X)$. Hence

$$
\lim _{\alpha}\left(P_{\sigma} Q_{\sigma}\right)_{(m)}\left(u_{\alpha}\right)=\psi\left(P_{\sigma} Q_{\sigma}\right)=0
$$

for every fixed $\sigma$. Thus $\psi(P Q)=0$. On the other hand, $\psi(P) \psi(Q)=0$ by the definition of $\psi$. So $\psi(P Q)=\psi(P) \psi(Q)$.

Thus we have defined the multiplicative function $\psi$ on homogeneous polynomials. We can extend it by linearity and distributivity to a linear multiplicative functional on the algebra of all continuous polynomials $\mathcal{P}(X)$. If $\psi_{n}$ is the restriction of $\psi$ to $\mathcal{P}\left({ }^{n} X\right)$, then $\left\|\psi_{n}\right\|=\|w\|^{n / m}$ if $n / m$ is a positive integer and $\left\|\psi_{n}\right\|=0$ otherwise. Hence $\psi=\sum_{n=0}^{\infty} \psi_{n}$ is a continuous linear multiplicative functional on $H_{b}(X)$ by [3. 2.4. Theorem] and the radius function of $\psi$ can be computed by

$$
R(\psi)=\limsup _{n \rightarrow \infty}\left\|\psi_{n}\right\|^{1 / n}=\limsup _{n \rightarrow \infty}\|w\|^{n / m n}=\|w\|^{1 / m}=\left\|\phi_{m}\right\|^{1 / m}
$$

as required.

For each fixed $x \in X$, the translation operator $T_{x}$ is defined on $H_{b}(X)$ by

$$
\left(T_{x} f\right)(y)=f(y+x), \quad f \in H_{b}(X) .
$$

It is not complicated to check that $T_{x} f \in H_{b}(X)$ and for fixed $\phi \in H_{b}(X)^{\prime}$ the function $x \mapsto \phi\left(T_{x} f\right), x \in X$, belongs to $H_{b}(X)$ (see [3]).

For fixed $\phi, \theta \in H_{b}(X)^{\prime}$ the convolution product $\phi * \theta$ in $H_{b}(X)$ is defined by

$$
(\phi * \theta)(f)=\phi\left(\theta\left(T_{x} f\right)\right), \quad f \in H_{b}(X) .
$$

Let $\phi, \theta \in M_{b}$. According to [3, 4.7. Corollary], there exist nets $\left(x_{\alpha}\right),\left(y_{\beta}\right) \subset X$ such that

$$
\phi(P)=\lim _{\alpha} P\left(x_{\alpha}\right), \quad \theta(P)=\lim _{\beta} P\left(y_{\beta}\right)
$$

for every polynomial $P$. We will write the condition (2) by $x_{\alpha} \stackrel{\mathcal{P}}{\rightarrow} \phi$ and $y_{\beta} \stackrel{\mathcal{P}}{\rightarrow} \theta$. Thus for every polynomial $P$ we have: $(\phi * \theta)(P)=\lim _{\beta} \lim _{\alpha} P\left(x_{\alpha}+y_{\beta}\right)$. Note that $M_{b}$ is a semigroup with respect to the convolution product and $\phi * \theta \neq \theta * \phi$ in general (see [5, Remark 3.5]). We denote $\phi_{1} * \cdots * \phi_{n}$ briefly by $\underset{k=1}{n} \phi_{k}$.

Let $I_{k}$ be the minimal closed ideal in $H_{b}(X)$, generated by all $m$-homogeneous polynomials, $0<m \leq k$. Evidently, $I_{k}$ is a proper ideal (contains no unit) so it is contained in a closed maximal ideal (see [21, p. 228]). Let

$$
\Phi_{k}:=\left\{\phi \in M_{b}: \operatorname{ker} \phi \supset I_{k}\right\} .
$$

We set $\Phi_{0}:=M_{b}$. The functional $\delta(0)$, that is, point evaluation at zero, belongs to $\Phi_{k}$ for every $k>0$. 
Corollary 2. If $A_{m}(X) \neq A_{m-1}(X)$ for some $m>1$, then there exists $\psi \in \Phi_{m-1}$ such that $\psi \notin \Phi_{m}$.

Proof. Let $P \in \mathcal{P}\left({ }^{m} X\right)$ and $P \notin A_{m-1}(X)$. Since $A_{m-1}(X)$ is a closed subspace of $H_{b}(X)$, by the Hahn-Banach Theorem there exists a linear functional $\phi \in H_{b}(X)^{\prime}$ such that $\phi(Q)=0$ for every $Q \in A_{m-1}(X)$ and $\phi(P) \neq 0$. So $\phi_{k} \equiv 0$ for $k<m$ and $\phi_{m}(P) \neq 0$. By Lemma 1 there exists $\psi \in M_{b}$ such that $\psi_{k}=\phi_{k}$ for $k=1, \ldots, m$. Thus $\psi \in \Phi_{m-1}$, but $\psi \notin \Phi_{m}$.

Note that $A_{1}\left(c_{0}\right)=A_{n}\left(c_{0}\right)$ for every $n$, but $A_{k}\left(\ell_{p}\right)=A_{m}\left(\ell_{p}\right)$ for $k \neq m$ if and only if $k<p$ and $m<p$. Moreover, if $X$ admits a polynomial which is not weakly sequentially continuous, then the chain of algebras $\left\{A_{k}(X)\right\}$ does not stabilize and if $X$ contains $\ell_{1}$, then $A_{k}(X) \neq A_{m}(X)$ for $k \neq m$ [19, 12].

Lemma 3. If $\phi, \psi \in M_{b}$ and $\psi \in \Phi_{k-1}$, then $\phi * \psi(P)=\phi(P)+\psi(P)$ for every $P \in \mathcal{P}\left({ }^{k} X\right)$.

Proof. Let $\left(x_{\alpha}\right)$ and $\left(y_{\beta}\right)$ be nets in $X$ such that $x_{\alpha} \stackrel{\mathcal{P}}{\rightarrow} \phi$ and $y_{\beta} \stackrel{\mathcal{P}}{\rightarrow} \psi$. For any fixed $y_{\beta}$ and $0<n<k, A_{P}\left(x^{k-n}, y_{\beta}^{n}\right)$ is a $(k-n)$-homogeneous polynomial. Thus

$$
\phi\left(A_{P}\left(x^{k-n}, y_{\beta}^{n}\right)\right)=\lim _{\alpha} A_{P}\left(x_{\alpha}^{k-n}, y_{\beta}^{n}\right)=0 .
$$

Therefore,

$$
\begin{aligned}
\phi * \psi(P) & =\lim _{\beta, \alpha} P\left(x_{\alpha}+y_{\beta}\right) \\
& =\sum_{n+m=k} \lim _{\beta, \alpha} A_{P}\left(x_{\alpha}^{n}, y_{\beta}^{m}\right)=\sum_{n+m=k} \lim _{\beta}\left(\lim _{\alpha} A_{P}\left(x_{\alpha}^{n}, y_{\beta}^{m}\right)\right) \\
& =\lim _{\beta}\left(\lim _{\alpha} A_{P}\left(x_{\alpha}, \ldots, x_{\alpha}\right)+A_{P}\left(y_{\beta}, \ldots, y_{\beta}\right)\right)=\phi(P)+\psi(P) .
\end{aligned}
$$

Lemma 4. If $P \in \mathcal{P}\left({ }^{k} X\right), \phi_{j} \in \Phi_{j-1}$, then for every $m>k, \underset{j=1}{\stackrel{m}{*}} \phi_{j}(P)=\underset{j=1}{*} \phi_{j}(P)$.

Proof. Since $\phi_{j} \in \Phi_{j-1}, \phi_{j}(P)=0$ for every $j>k$.

Given a sequence $\left(\phi_{n}\right)_{n=1}^{\infty} \subset M_{b}, \phi_{n} \in \Phi_{n-1}$, the infinite convolution $\underset{n=1}{*} \phi_{n}$ denotes a linear multiplicative functional on the algebra of all polynomials $\mathcal{P}(X)$ such that $\underset{n=1}{\stackrel{\infty}{*}} \phi_{n}(P)=\underset{n=1}{*}(P)$ if $P \in \mathcal{P}\left({ }^{k} X\right)$ for an arbitrary $k$. This multiplicative functional uniquely determines a functional in $M_{b}$ (which we denote by the same symbol $\left.\underset{n=1}{\stackrel{*}{*}} \phi_{n}\right)$ if it is continuous.

The point evaluation operator $\delta$ maps $X$ into $M_{b}$ by $x \mapsto \delta(x), \delta(x)(f)=f(x)$. The operator $\widetilde{\delta}$ is the extension of $\delta$ onto $X^{\prime \prime}$, i.e. $\widetilde{\delta}\left(x^{\prime \prime}\right)(f)=\widetilde{f}\left(x^{\prime \prime}\right)$ for every $x^{\prime \prime} \in X^{\prime \prime}$.

Theorem 5. There exists a sequence of dual Banach spaces $\left(E_{n}\right)_{n=1}^{\infty}$ and a sequence of maps $\delta^{(n)}: E_{n} \rightarrow M_{b}$ such that $E_{1}=X^{\prime \prime}, E_{n}=\mathcal{P}\left({ }^{n} X\right)^{\prime} \cap I_{n-1}^{\perp}, \delta^{(1)}=\tilde{\delta}$ and such that an arbitrary complex homomorphism $\phi \in M_{b}$ has a representation

$$
\phi=\underset{n=1}{\infty} \delta^{(n)}\left(u_{n}\right)
$$

for some $u_{n} \in E_{n}, n=1,2, \ldots$ 
Proof. Put $E_{1}=X^{\prime \prime}$. Then $\delta^{(1)}\left(x^{\prime \prime}\right)=\tilde{\delta}\left(x^{\prime \prime}\right) \in M_{b}$ for every $x^{\prime \prime} \in X^{\prime \prime}$. Suppose that spaces $E_{k}$ and maps $\delta^{(k)}$ are constructed for $k<n$. Denote by $E_{n}$ the set $\left\{\pi_{n}(\phi): \phi \in \Phi_{n-1}\right\}$, where $\pi_{n}(\phi)=\phi_{n}$ is the restriction of $\phi$ onto the subspace $\mathcal{P}\left({ }^{n} X\right)$. In other words, $E_{n}$ consists of linear continuous functionals on $\mathcal{P}\left({ }^{n} X\right)$ that vanish on all polynomials in $\mathcal{P}\left({ }^{n} X\right) \cap A_{n-1}$. If $A_{n}=A_{n-1}$, then $E_{n} \equiv 0$. Otherwise, by Corollary 2 there are nonzero points in $E_{n}$.

By Lemma 3, for $P \in \mathcal{P}\left({ }^{n} X\right)$ and $\phi, \psi \in \Phi_{n-1} \subset M_{b}, \pi_{n}(\phi * \psi)(P)=\phi * \psi(P)=$ $\phi(P)+\psi(P)=\pi_{n} \phi(P)+\pi_{n} \psi(P)$. Hence $\pi_{n}(\phi * \psi)=\pi_{n}(\phi)+\pi_{n}(\psi)$. For an arbitrary complex number $a, a \phi \in H_{b}(X)^{\prime}$ and $\pi_{k}(a \phi)=a \pi_{k}(\phi)$. So $a \phi$ vanishes on all homogeneous polynomials of degree less than $n$. By Lemma 1 there exists $\psi \in M_{b}$ such that $\psi_{k}=a \phi_{k}$ for $1 \leq k \leq n$. Thus $\psi \in \Phi_{n-1}$ and $a \phi_{n}=\psi_{n} \in E_{n}$. Hence $E_{n}$ is a linear space and polynomials from $\mathcal{P}\left({ }^{n} X\right)$ are acting on $E_{n}$ as linear functionals. Put $W_{n}=\mathcal{P}\left({ }^{n} X\right) /\left(I_{n-1} \cap \mathcal{P}\left({ }^{n} X\right)\right)$. Then $W_{n}$ is a Banach space of linear functionals on $E_{n}$ and the functionals from $W_{n}$ separate points of $E_{n}$. Let us define a norm on $E_{n},\|\cdot\|_{n}$ as the supremum of values of a vector from $E_{n}$ on the unit ball of $W_{n}$. Therefore $W_{n}^{\prime}=\left(\mathcal{P}\left({ }^{n} X\right) /\left(I_{n-1} \cap \mathcal{P}\left({ }^{n} X\right)\right)\right)^{\prime}=\mathcal{P}\left({ }^{n} X\right)^{\prime} \cap I_{n-1}^{\perp} \supset E_{n}$. On the other hand, if $u \in \mathcal{P}\left({ }^{n} X\right)^{\prime} \cap I_{n-1}^{\perp}$, then by Lemma 1 $u=\pi_{n}(\phi)$ for some $\phi \in M_{b}$ and so $u \in E_{n}$. Thus $E_{n}=W_{n}^{\prime}$.

For given $w \in E_{n}$ let us define $\delta^{(n)}(w)(Q)=\psi(Q)$ on homogeneous polynomials $Q$ by formula (11) and extend it to the unique complex homomorphism on $H_{b}(X)$ as in Lemma 1. So $\delta^{(n)}$ maps $E_{n}$ into $M_{b}$. For any $\phi \in M_{b}$ put $u_{1}:=\phi_{1} \in X^{\prime \prime}=E_{1}$, $u_{2}:=\phi_{2}-\pi_{2}\left(\delta^{(1)}\left(u_{1}\right)\right)$. It is clear that $u_{2} \in E_{2}$. Suppose that we have defined $u_{k} \in E_{k}, k<n$. Set

$$
u_{n}:=\phi_{n}-\pi_{n}\left(\begin{array}{l}
n-1 \\
k=1
\end{array} \delta^{(k)}\left(u_{k}\right)\right) .
$$

Let us show that $u_{n} \in E_{n}$. It is enough to check that for every $P \in \mathcal{P}\left({ }^{n} X\right)$ such that $P=P_{k} P_{m}, \operatorname{deg} P_{k}=k \neq 0, \operatorname{deg} P_{n}=n \neq 0$ implies $u_{n}(P)=0$. Note that for all $n$-homogeneous polynomials $P_{n}$,

$$
\phi_{n}-\pi_{n}\left(\begin{array}{l}
n-1 \\
k=1 \\
k=1
\end{array} \delta^{(k)}\left(u_{k}\right)\right)\left(P_{n}\right)=\phi_{n}-\underset{k=1}{n-1} \delta^{(k)}\left(u_{k}\right)\left(P_{n}\right) .
$$

From the multiplicativity of $\phi$ and Lemma 4 it follows that

$$
\begin{aligned}
& \left.u_{n}(P)=\phi_{n}\left(P_{k} P_{m}\right)-{\stackrel{\text { w }}{*} \delta^{n-1} \delta^{(j)}}_{\left(u_{j}\right.}\right)\left(P_{k} P_{m}\right)=\phi_{k}\left(P_{k}\right) \phi_{m}\left(P_{m}\right)
\end{aligned}
$$

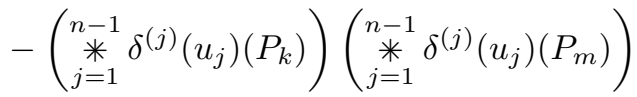

$$
\begin{aligned}
& =\left(u_{k}\left(P_{k}\right)+\underset{j=1}{\stackrel{k}{*} \delta^{(j)}}\left(u_{j}\right)\left(P_{k}\right)\right)\left(u_{m}\left(P_{m}\right)+\underset{j=1}{\stackrel{m-1}{*} \delta^{(j)}}\left(u_{j}\right)\left(P_{m}\right)\right) \\
& -\left(\underset{j=1}{\stackrel{*}{*} \delta^{(j)}}\left(u_{j}\right)\left(P_{k}\right)\right)\left(\underset{j=1}{\stackrel{m}{*}} \delta^{(j)}\left(u_{j}\right)\left(P_{m}\right)\right)=0 .
\end{aligned}
$$

The last equality holds because by the induction assumption, $u_{k} \in E_{k}, u_{m} \in E_{m}$ and hence, by Lemma 3 ,

$$
u_{k}\left(P_{k}\right)+\underset{j=1}{k-1} \delta^{(j)}\left(u_{j}\right)\left(P_{k}\right)=\underset{j=1}{*} \delta^{(j)}\left(u_{j}\right)\left(P_{k}\right)
$$


and

$$
u_{m}\left(P_{m}\right)+\underset{j=1}{\stackrel{m-1}{*} \delta^{(j)}}\left(u_{j}\right)\left(P_{m}\right)=\underset{j=1}{m} \delta^{(j)}\left(u_{j}\right)\left(P_{m}\right) .
$$

Let us consider the functional $\underset{j=1}{\infty} \delta^{(j)}\left(u_{j}\right)$. Since $u_{k} \in E_{k}$, by Lemma 3 ,

$$
\underset{j=1}{\stackrel{*}{*} \delta^{(j)}}\left(u_{j}\right)(f)=f(0)+\sum_{n=1}^{\infty} \underset{j=1}{*} \delta^{(j)}\left(u_{j}\right)\left(f_{n}\right)
$$

where $f=\sum f_{n}$ is the Taylor series expansion of $f$. Hence $\underset{j=1}{\infty} \delta^{*}(j)\left(u_{j}\right)$ is well defined on $\mathcal{P}(X)$. On the other hand, applying (4) and (5) we obtain

$$
\begin{aligned}
\left(\phi-\underset{j=1}{*} \delta^{(j)}\left(u_{j}\right)\right)\left(P_{n}\right) & =\phi_{n}\left(P_{n}\right)-\underset{j=1}{*} \delta^{(j)}\left(u_{j}\right)\left(P_{n}\right) \\
& =u_{n}(P)+\underset{j=1}{n-1} \delta^{(j)}\left(u_{j}\right)\left(P_{n}\right)-\underset{j=1}{*} \delta^{*} \delta^{(j)}\left(u_{j}\right)\left(P_{n}\right)=0
\end{aligned}
$$

for arbitrary $P_{n} \in \mathcal{P}\left({ }^{n} X\right)$. Thus $\phi=\underset{j=1}{\substack{* \\ j=1}} \delta^{(j)}\left(u_{j}\right)$ on $\mathcal{P}(X)$. Hence $\phi=\underset{j=1}{\stackrel{*}{*}} \delta^{(j)}\left(u_{j}\right)$ on $H_{b}(X)$.

Let us denote by $E^{\infty}$ the space of all finite sequences $\left(u_{1}, \ldots, u_{m}, 0, \ldots\right), u_{k} \in E_{k}$. According to Theorem [5, every finite sequence $\mathfrak{u}=\left(u_{1}, \ldots, u_{m}, 0, \ldots\right)$ defines a character $\phi_{\mathfrak{u}}=\underset{k=1}{m} \delta^{(k)}\left(u_{k}\right) \in M_{b}$. Thus $E^{\infty} \subset M_{b}$ and for every $\mathfrak{u}, \mathfrak{v} \in E^{\infty}$, $\phi_{\mathfrak{u}+\mathfrak{v}} \in M_{b}$. Moreover, from the density of polynomials in $H_{b}(X)$ it follows that $E^{\infty}$ is dense in $M_{b}$ with respect to the Gelfand topology. So we have proved the following theorem.

Theorem 6. $M_{b}$ contains the dense linear subspace of all finite subsequences $\left(u_{1}, \ldots, u_{m}, 0, \ldots\right), u_{k} \in E^{k}$.

According to [3, 7, the operation of sum on $X$ may be discontinuous with respect to the Gelfand topology, induced from $M_{b}$. Hence, in general, $E^{\infty}$ is not a topological vector space. Thus, the density of $E^{\infty}$ in $M_{b}$ does not imply that $M_{b}$ is a linear space.

We need to have some properties of the radius function, proved by Aron, Cole and Gamelin in 3 .

Proposition 7. (1) For each $r>0$, the set of $\phi \in M_{b}$ satisfying $R(\phi) \leq r$ coincides with the spectrum of $H_{u c}^{\infty}(r B)$. In particular, $M_{u c}=\left\{\phi \in M_{b}\right.$ : $R(\phi) \leq 1\}$

(2) For every $\phi, \psi \in H_{b}(x)^{\prime}, R(\phi * \psi) \leq R(\phi)+R(\psi)$.

Example 8. 1. Let $X$ be $c_{0}$ or Tsirelson's space. Then $E_{k}=\{0\}$ for $k>1$ [4, 22].

2. Let $X=\ell_{1}$ and $\phi \in H_{b}\left(\ell_{1}\right)^{\prime},\|\phi\|=1$. According to [3], $\phi \in M_{b}\left(\ell_{1}\right)$ if and only if for every $m=1,2, \ldots$ there exists a symmetric measure on $\beta\left(\mathbb{N}^{m}\right), \nu_{m}$ and a constant $c>0$ such that $\left\|\nu_{m}\right\| \leq c^{m}$ and for each $P_{m} \in \mathcal{P}\left({ }^{m} \ell_{1}\right)$,

$$
\phi\left(P_{m}\right)=\int_{\beta(\mathbb{N} m)} \widehat{P}_{m} d \nu_{m}
$$


where $\widehat{P}_{m}$ is just $P_{m}$ regarded as a vector from $\ell^{\infty}\left(\mathbb{N}^{m}\right)$. By Theorem $5 \phi \in M_{b}\left(\ell_{1}\right)$ if and only if there is a sequence of symmetric measures $\left(\mu_{m}\right)$ which are orthogonal to $\beta\left(\mathbb{N}^{j}\right) \times \beta\left(\mathbb{N}^{k}\right) \subset \beta\left(\mathbb{N}^{m}\right)$, for $m>1, k+j=m, k, j>0$, and functionals

$$
u_{m}\left(P_{m}\right)=\int_{\beta\left(\mathbb{N}^{m}\right)} \widehat{P}_{m} d \mu_{m}
$$

determine $\phi$ by formula (3).

3. (Cf. [1, Example 3.1].) Let $X=\ell_{p}$ for some integer $p, 1<p<\infty$. For every $n$, put

$$
v_{n}=\frac{1}{n^{1 / p}}\left(e_{1}+\cdots+e_{n}\right),
$$

where $\left(e_{k}\right)$ is the standard basis in $\ell_{p}$. Since $\left\|v_{n}\right\|=1, R\left(\delta\left(v_{n}\right)\right)=1$ and so $\delta\left(v_{n}\right) \in M_{u c} \subset M_{b}$. By compactness of $M_{u c}$ there is an accumulation point $\phi \in M_{u c}$ of the sequence $\left(\delta\left(v_{n}\right)\right)$. If $0<k<p$, then by Pitt's Theorem (see [16, Theorem 5.1]) every polynomial $P \in \mathcal{P}\left({ }^{k} \ell_{p}\right)$ is weakly continuous on bounded sets. Since $v_{n}$ is weakly null in $\ell_{p}, \phi(P)=0$. On the other hand, $\phi(Q)=1$ for the polynomial $Q(x)=\sum_{n=1}^{\infty} x_{n}^{p}$. Thus $\phi \in \Phi_{p-1}$ and $\phi \neq 0$. In other words, if $\phi=\underset{k=1}{\infty} \delta^{(k)}\left(u_{k}\right)$ is the representation of $\phi$ by Theorem 5 , then $u_{k}=0$ for $k<p$ and $u_{p} \neq 0$.

Proposition 9. Let $\phi \in M_{b}$ and let $\phi=\underset{k=1}{\stackrel{\infty}{*}} \delta^{(k)}\left(u_{k}\right), u_{k} \in E_{k}$, be its representation. Then

$$
\limsup _{k \rightarrow \infty}\left\|u_{k}\right\|_{k}^{1 / k} \leq R(\phi) \leq \sum_{k=1}^{\infty}\left\|u_{k}\right\|_{k}^{1 / k} .
$$

Proof. The first inequality holds because $\left\|u_{k}\right\|_{k} \leq\left\|\phi_{k}\right\|$ and by the definition of the radius function. The second inequality follows from Proposition 7 and the following calculation:

$$
R\left(\delta^{(k)}\left(u_{k}\right)\right)=\limsup _{m \rightarrow \infty}\left\|\pi_{k m}\left(\delta^{(k)}\left(u_{k}\right)\right)\right\|^{1 / k m}=\left\|\delta^{(k)}\left(u_{k}\right)\right\|^{m / k m}=\left\|u_{k}\right\|^{1 / k} .
$$

Let $F$ be an analytic map from $\ell_{1}$ to $\ell_{1}$ defined by

$$
F(x)=F\left(\sum_{n=1}^{\infty} x_{n} e_{n}\right)=\sum_{n=1}^{\infty} x_{n}^{n} e_{n} .
$$

We denote by $F\left(\ell_{1}\right)$ the range of $F$ and by $F\left(B_{\ell_{1}}\right)$ the range of $F$ restricted to the unit ball $B_{\ell_{1}} \subset \ell_{1}$.

Given a sequence of Banach spaces $\left(E_{n},\|\cdot\|_{n}\right)_{n=1}^{\infty}$ and $0<\rho \leq \infty$ the Köthe sequence space $\lambda^{1}\left(K_{\rho} ;\left(E_{n}\right)\right.$ ) (where $K_{\rho}=\left\{\left(r^{n}\right)_{n=1}^{\infty}: 0<r<\rho\right\}$ ) is the Fréchet space

$$
\left\{\left(x_{n}\right)_{n=1}^{\infty} \in \prod_{n=1}^{\infty} E_{n}: p_{r}\left(\left(x_{n}\right)_{n=1}^{\infty}\right)=\sum_{n=1}^{\infty}\left\|x_{n}\right\| r^{n}<\infty \forall r, 0<r<\rho\right\},
$$

endowed with the topology given by the seminorms $\left\{p_{r}\right\}_{0<r<\rho}$. By CauchyHadamard's formula,

$$
\lambda^{1}\left(K_{\rho} ;\left(E_{n}\right)\right)=\left\{\left(x_{n}\right)_{n=1}^{\infty} \in \prod_{n=1}^{\infty} E_{n}: \limsup _{n \rightarrow \infty}\left\|x_{n}\right\|_{n}^{1 / n} \leq \frac{1}{\rho}\right\} .
$$


Corollary 10. (1) $M_{b}$ contains every sequence $\mathfrak{u}=\left(u_{k}\right)_{k=1}^{\infty}, u_{k} \in E_{k}$, such that the sequence $\left(\left\|\left(u_{k}\right)\right\|\right)_{k=1}^{\infty}$ is in $F\left(\ell_{1}\right)$.

(2) $M_{u c}$ contains every sequence $\mathfrak{u}=\left(u_{k}\right)_{k=1}^{\infty}, u_{k} \in E_{k}$, such that the sequence $\left(\left\|\left(u_{k}\right)\right\|\right)_{k=1}^{\infty}$ is in $F\left(B_{\ell_{1}}\right)$.

(3) Every complex homomorphism $\phi \in M_{b}$ is contained in a Köthe sequence space $\lambda^{1}\left(K_{\rho} ;\left(E_{n}\right)\right)$ for $\rho=1 / R(\phi)$.

(4) $M_{u c}$ is contained in $\lambda^{1}\left(K_{1} ;\left(E_{n}\right)\right)$.

Proof. Since $F^{-1}\left(\left(\left\|u_{k}\right\|\right)_{k=1}^{\infty}\right) \in \ell_{1}, \sum_{k=1}^{\infty}\left\|u_{k}\right\|^{1 / k} \leq \infty$ and by Proposition 9, $R\left(\phi_{\mathfrak{u}}\right)<\infty$. Thus $\phi_{\mathfrak{u}} \in M_{b}$. Moreover, if $F^{-1}\left(\left(\left\|u_{k}\right\|\right)_{k=1}^{\infty}\right) \in F\left(B_{\ell_{1}}\right)$, then $R\left(\phi_{\mathfrak{u}}\right) \leq 1$ and $\phi_{\mathfrak{u}} \in M_{u c}$.

Suppose that $\phi_{\mathfrak{u}} \in M_{b}$ for some $\mathfrak{u}=\left(u_{k}\right)_{k=1}^{\infty}$. Then $R\left(\phi_{\mathfrak{u}}\right)<\infty$ and by Proposition 9. $\limsup _{k \rightarrow \infty}\left\|u_{k}\right\|^{1 / k} \leq R\left(\phi_{\mathfrak{u}}\right)$. Hence $\phi_{\mathfrak{u}} \in \lambda^{1}\left(K_{1 / R\left(\phi_{\mathfrak{u}}\right)} ;\left(E_{n}\right)\right)$. In particular, if $R\left(\phi_{\mathfrak{u}}\right) \leq 1$, then $\phi_{\mathfrak{u}} \in \lambda^{1}\left(K_{1} ;\left(E_{n}\right)\right)$.

Dixon 14 has given an example of an algebra of polynomials of infinitely many variables which admits discontinuous scalar-valued homomorphisms. In [15] a construction is given of a discontinuous scalar-valued homomorphism of an algebra of polynomials on an arbitrary infinite-dimensional Banach space. The next corollary shows that the restriction of a discontinuous complex homomorphism on $A_{n}(X) \cap \mathcal{P}(X)$ can be continuous for every $n$. Note that the problem of existence of discontinuous complex homomorphisms on $H_{b}(X)$ for an infinitele-dimensional Banach space $X$ is still open and equivalent to the famous Michael Problem [20], [21, p. 240].

Corollary 11. If the sequence of algebras $A_{n}(X)$ does not stabilize, then there is a discontinuous complex homomorphism $\zeta$ on $\mathcal{P}(X)$ such that the restriction of $\zeta$ on $A_{n}(X) \cap \mathcal{P}(X)$ is a continuous complex homomorphism for every $n$.

Proof. By Corollary 2 and Theorem 5 there exists an infinite sequence $\left(u_{k}\right)_{k=1}^{\infty}$, $u_{k} \in E_{k}, u_{k} \neq 0$. Since each $E_{k}$ is a linear space, we can choose $u_{k}$ such that

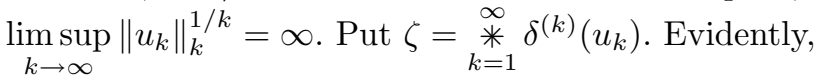

$$
\zeta(f)=\underset{k=1}{*} \delta^{(k)}\left(u_{k}\right)(f)
$$

for every $f \in A_{n}(X)$. So $\zeta$ is well defined and continuous on $A_{n}(X) \cap \mathcal{P}(X)$. If $\zeta$ is continuous on $\mathcal{P}(X)$, then it can be extended to a continuous complex homomorphism on $H_{b}(X)$. But this contradicts Proposition 9 .

In 11 Deghoul, using Borsuk's theorem, shows that there is an "exceptional" character $\phi$ on $H_{b}\left(\ell_{2}\right)$ such that $\phi$ vanishes on odd degree homogeneous polynomials and is different from the evaluation at 0 . The next proposition delivers the existence of exceptional characters on $H_{b}(X)$ for a large number of $X$.

Proposition 12. Suppose that $A_{m}(X) \neq A_{k}(X)$ for some $m>1$ and all $k<m$. Then there exists a nontrivial character $\psi_{0} \in M_{b}$ such that $\psi_{0}(P)=0$ for every homogeneous polynomial $P, \operatorname{deg} P \neq n m, n=1, \ldots, \infty$. 
Proof. By Corollary 2 there exists a nontrivial character $\psi \in M_{b}$ which vanishes on all $k$-homogeneous polynomials for $k<m$. From Theorem 5 it follows that $E_{m}$ contains a nonzero vector $u_{m}$. Put $\psi_{0}=\delta^{(m)}\left(u_{m}\right)$. Then $\psi_{0}$ vanishes on all homogeneous polynomials excepting $n m$-homogeneous polynomials, $n=1,2, \ldots$.

\section{ACKNOWLEDGEMENTS}

The author is greatly indebted to the referee for his attention to the paper and numerous helpful comments. The author wishes to thank the University of Saskatchewan, where the paper was written, for the invitation and financial support.

\section{REFERENCES}

1. R. Alencar, R. Aron, P. Galindo, and A. Zagorodnyuk, Algebras of symmetric holomorphic functions on $\ell_{p}$, Bull. Lond. Math. Soc. 35 (2003), 55-64. MR.1934432 (2003m:46082)

2. R.M. Aron and P.D. Berner, A Hahn-Banach extension theorem for analytic mappings, Bull. Soc. Math. France 106 (1978), 3-24. MR0508947 (80e:46029)

3. R.M. Aron, B.J. Cole, and T.W. Gamelin, Spectra of algebras of analytic functions on a Banach space, J. Reine Angew. Math. 415 (1991), 51-93. MR1096902 (92f:46056)

4. R.M. Aron, B.J. Cole, and T.W. Gamelin, Weak-star continuous analytic funtions, Can. J. Math. 47 (1995), 673-683. MR.1346158 (96d:46060)

5. R.M. Aron, P. Galindo, D. Garcia, and M. Maestre, Regularity and algebras of analytic functions in infinite dimensions, Trans. Amer. Math. Soc. 348 (1996), 543-559. MR1340167 (96g:46032)

6. R.M. Aron and J.B. Prolla, Polynomial approximation of differentiable functions on Banach spaces, J. Reine Angew. Math. 313 (1980), 195-216. MR.0552473 (81c:41078)

7. P. Biström, J.A. Jaramillo, and M. Lindström, Polynomial compactness in Banach spaces, Rocky Mount. J. Math. 28 (1998), 1203-1225. MR.1681664 (2000c:46038)

8. F. Cabello Sánchez, R. García, and I. Villanueva, Extension of multilinear operators on Banach spaces, Extracta Math. 15 (2000), 291-334. MR.1823895 (2002e:46022)

9. D. Carando, D. García and M. Maestre, Homomorphisms and composition operators on algebras of analytic functions of bounded type, Adv. Math. To appear.

10. A.M. Davie and T.W. Gamelin, A theorem on polynomial-star approximation, Proc. Amer. Math. Soc. 106 (1989), 351-356. MR0947313 (89k:46023)

11. D. Deghoul, Construction de caractères exceptionnels sur une algèbre de Fréchet, C. R. Acad. Sci. Paris. 312 (1991), 579-580. MR1101036 (92b:46066)

12. V. Dimant and R. Gonzalo, Block diagonal polynomials, Trans. Amer. Math. Soc. 353 (2001), 733-747. MR 1804515 (2002b:46079)

13. S. Dineen, Complex Analysis on Infinite Dimensional Spaces, Monographs in Mathematics, Springer, New York, 1999. MR1705327 (2001a:46043)

14. P. Dixon, Scalar homomorphisms on algebras of infinitely long polynomials with an application to automatic continuity theory, J. London Math. Soc. (2) 19 (1979), 488-496. MR0540065 (80h:46074)

15. P. Galindo, D. García, M. Maestre, and J. Mujica, Extension of multilinear mappings on Banach spaces, Studia. Math. 108 (1994), 55-76. MR1259024 (95f:46072)

16. T.W. Gamelin, Analytic functions on Banach spaces, in Complex Potential Theory, Ed. Gauthier and Sabidussi, Kluwer Academic Publishers, Amsterdam, 1994, 187-233. MR 1332962 (96m:46082)

17. D. García, M.L. Lourenço, M. Maestre, and L.A. Moraes, The spectrum of analytic mappings of bounded type, J. Math. Anal. Appl. 245 (2000), 447-470. MR1758549 (2001f:46069)

18. D. García, M.L. Lourenço, L.A. Moraes, and O.W. Paques, The spectra of some algebras of analytic mappings, Indag. Mathem. 10 (1999), 393-406. MR1819897 (2002c:46089)

19. R. Gonzalo, Multilinear forms, subsymmetric polynomials, and spreading models on Banach spaces, J. Math. Anal. App. 202 (1996), 379-397. MR.1406236|(98a:46054)

20. E. Michael, Locally multiplicatively convex topological algebras, Mem. Amer. Math. Soc., vol. 11, Providence, 1952. MR0051444 (14:482a) 
21. J. Mujica, Complex Analysis in Banach Spaces, North-Holland, Amsterdam, New York, Oxford, 1986. MR0842435 (88d:46084)

22. J. Mujica, Ideals of holomorphic functions on Tsirelson's space, Archiv der Mathematik 76 (2001), 292-298. MR.1825009 (2002g:46075)

Institute for Applied Problems of Mechanics and Mathematics, Ukrainian Academy of Sciences, 3 B, NAukova str., LViv 79060, Ukraine

Current address: Department of Mathematics and Statistics, McLean Hall, University of Saskatchewan, 106 Wiggins Road, Saskatoon, Saskatchewan, Canada S7N 5E6

E-mail address: zahorodn@math.usask.ca

E-mail address: andriyzag@yahoo.com 